\title{
Production of an energy-storage electrode paper using a pilot-scale paper machine
}

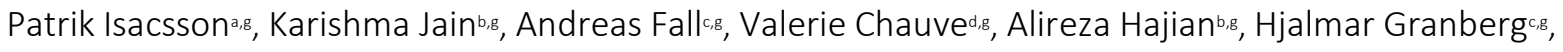 \\ Lucie Boiron d,s, Magnus Berggrena,f,g, Karl Håkanssonnc,s, Jesper Edberge,s, Isak Engquista,f,g, and Lars Wågbergb,g,h \\ a Laboratory of Organic Electronics, Department of Science and Technology, Linkoping University, 60174 Norrkoping, Sweden. \\ ${ }^{b}$ Department of Fibre and Polymer Technology, KTH Royal Institute of Technology, Teknikringen 56-58, SE-100 44 Stockholm, Sweden. \\ ${ }^{c} R I S E$ Research Institutes of Sweden, Bioeconomy and Health, SE-114 86 Stockholm, Sweden. \\ ${ }^{d}$ Ahlstrom-Munksjö Research Center, 38140 Apprieu, France. \\ ${ }^{e}$ RISE Research Institutes of Sweden, Digital Systems, Bio- and Organic Electronics, Bredgatan 35, Norrköping SE-602 21, Sweden. \\ fWallenberg Wood Science Center, Linköping University, Department of Science and Technology, Linkoping University, 60174 Norrkoping, Sweden. \\ ${ }^{g}$ Digital Cellulose Center (digitalcellulosecenter.se).

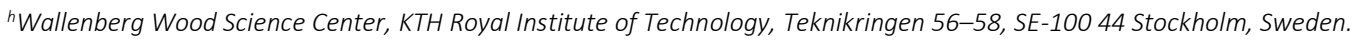

\begin{abstract}
The global electrification of our society requires an enormous capacity of electrical energy storage. This drives the demand for low-cost and sustainable solutions, where the electrode materials are key components. In the present work, all-organic supercapacitor electrodes have successfully been demonstrated to be produced on a pilot-scale paper machine, thereby showing the feasibility of largescale production of "paper-based energy storage". The material concept was based on activated charcoal from pyrolyzed coconut and cationized cellulose pulp, the latter having small amounts of electrostatically adsorbed PEDOT:PSS in order to create a conducting, percolating network. In a pre-trial lab experiment, it was evident that even small addition of $1 \mathrm{wt} \%$ PEDOT:PSS gave a large increase in capacitance compared to samples with only activated charcoal. In the pilot trials, the addition of carboxymethylated nanocellulose and/or carbon black was further investigated. The different additions significantly affected several paper properties such as tensile strength and conductivity, but the specific capacitance of the activated charcoal was not affected and was found to be around 65-70 F/g. As more than half of the electrodes mass consisted of pulp fibers, the specific capacitance of the paper electrodes was about 25-30 F/g, which is in the same order of commercial supercapacitor electrodes. The successful production of several 10-meter-long rolls of supercapacitor electrode paper shows the feasibility of producing energy storage devices with papermaking methods, and the work as a whole provides valuable insights on how to further advance bio-based energy storage solutions.
\end{abstract}

\section{Introduction}

The race towards replacing fossil fuels with renewable energy sources is accelerating due to a push from the public as well as the rapidly dropping prices in technologies such as solar cells. However, with these more sustainable technologies, new technical challenges have emerged. For a long time, the energy going into the power grid has been dominated by nuclear power and fossil fuels like oil, coal, and natural gas. Nuclear plants supply a relatively constant supply of power to the grid, as it is too costly to turn them on and off, which is something only done during maintenance. Instead, the fossil fuel-based power plants work as the regulators, producing more energy when demand is high and quickly reducing the production when demand is low. 
As fossil fuels are replaced with renewable energy sources (mainly wind and solar power), balancing the power grid is becoming a problem. After all, these new energy sources are intermittent, with the sun shining only in daytime and wind being highly irregular. While solar power is more predictable compared to wind (at least in areas with few cloudy days), a major issue is that they produce the maximum amount of power when demand is low, and little or no power when demand is high. In order to solve this problem, excess of produced energy needs to be stored to be used later when demand is high. While there are many alternatives to such intermittent energy storage, like pumped-storage hydroelectricity or flywheel energy storage, today the best and most versatile method is by electrochemical devices such as batteries and supercapacitors.

So far, traditional batteries have been used for industrial and grid energy storage, with lithium-ion batteries (LIB) dominating the market. But LIB have several inherent limitations such a high cost, short lifetime, poor safety and environmental hazards. The risk of thermal runaway, combustion, and even explosions caused by LIBs poses a serious risk, especially when considering using thousands of cells together which will be required for large scale energy storage. Also, lithium requires heavy mining for extraction which is becoming a problem in the countries where it originates from. But the greatest challenge might be still to come, as the batteries gets old and needs to be disposed of. As of today, there are no good ways of recycling LIBs, and so they will likely contribute to a mountain of electronic waste in the future. And so, there is a need to re-think battery technology to make it more sustainable.

In the last decade, several new battery technologies have emerged which used bio-based materials both as structural components and as the redox-active components (Wang et al 2020). These devices, sometimes called paper batteries, have been designed to be more environmentally friendly, both with respect to the material origin and to their disposal at end of life. Paper-based technologies also have potential for large scale production of electronics, as paper is produced in the hundreds of metric tons every year. While there are plenty of examples of such technologies in scientific literature, most studies have focused on lab-scale production, and there are few (if any) examples of scaled up production of such electronic paper. To become industrially relevant, new technologies needs to first take the step from labscale production to a pilot production, which for materials usually means going from the gram-scale to kilogram-scale. This important step is what has kept many promising technologies from reaching the market. One such example is graphene, which was first produces in 2004, but has just recently reached the market due to breakthroughs in scaled up manufacturing (Bubnova 2021). In this work, we have taken a similar step in the area of paper electronics by the production of a bio-based "energy-storage paper" in a pilot-scale paper machine.

Transferring material concepts designed for printing into papermaking conditions is not, however, that straightforward. Using nanocelluloses as the web-forming material alone is simply not possible due to its small size and water retention capacity (Peng et al 2012), why exchanging the nanoscale cellulose fibrils into macroscale pulp fibers would make the transition easier. The forming section in papermaking, where the fiber suspension is drained over a coarse wire, further put demands on a well-designed system in order to retain the materials to be included in the web. Furthermore, one of the greatest challenges in production of electronic paper is to create an electronically conductive network to efficiently transfer electrons to the charge-storing particles (in this work activated charcoal). In previous work, conductive polymers, such as poly(3,4-ethylenedioxythiophene) polystyrene sulfonate (PEDOT:PSS), have been demonstrated to work excellently for this purpose (Belaineh et al 2020). However, while labscale production of such composites has relied on methods like solution casting (Malti et al 2016), spray 
coating (Say et al 2020), or stencil printing (Brooke et al 2019), which requires relatively long drying times and thereby slow production rate; the step towards the fast dewatering rates used in papermaking requires new innovative solutions. The previous attempts of adding PEDOT:PSS to a papermaking process has been hindered by the inability of the nanosized particles to adhere to the cellulose fibers/fibrils in solution form. And so, when the mixture passes though a wire mesh or other filter, the fiber stick while the conductive polymer passes through with the water.

In this project, we have investigated the use of dissolving cellulose fibers, which upon chemical modification has been functionalized with cationic quaternary trimethylammonium groups (fig. 1). This addition of fixed positive charged groups on the fibers enabled electrostatic adsorption of negatively charged PEDOT:PSS nanoparticles to create a percolating conducting network between the active material components. This novel method solves the previous issues with adhesion of the conductive polymer to the fiber network and enables fast dewatering rates which are necessary for scaled up production. Furthermore, we investigate the effect of the addition of cellulose nanofibrils (CNF) and conductive carbon particles (carbon black), as well as different processing parameters, on the properties of the obtained paper such as mechanical strength, conductivity, and electrochemical charge storage. Finally, we use one of the more promising material combinations to assemble a full supercapacitor device.

a)

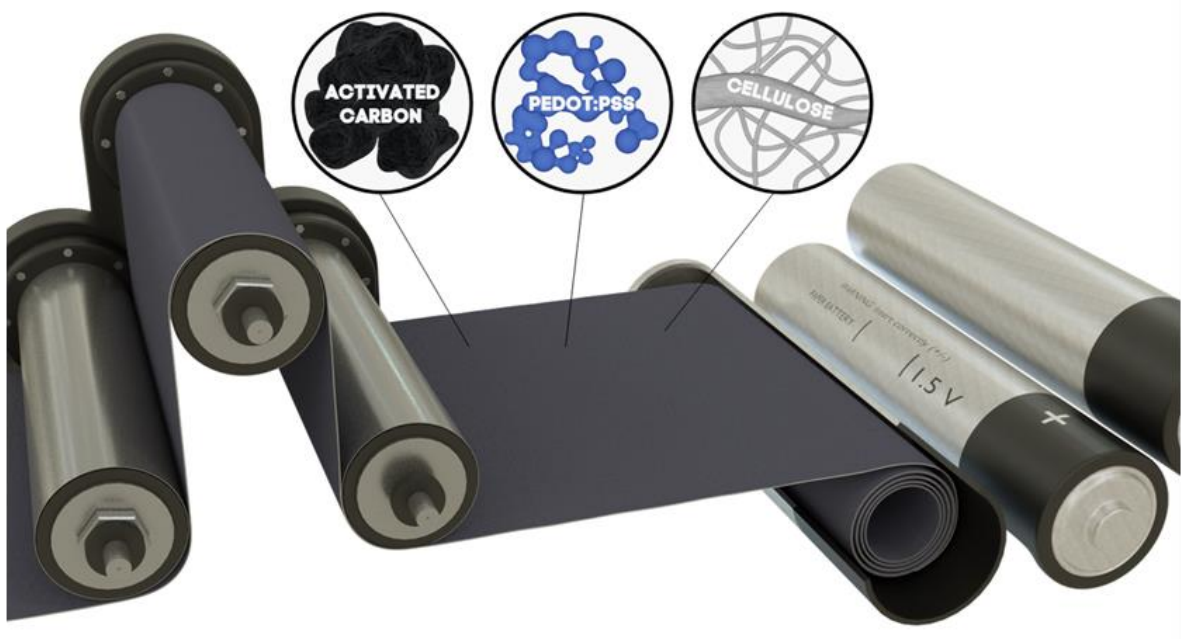

b)
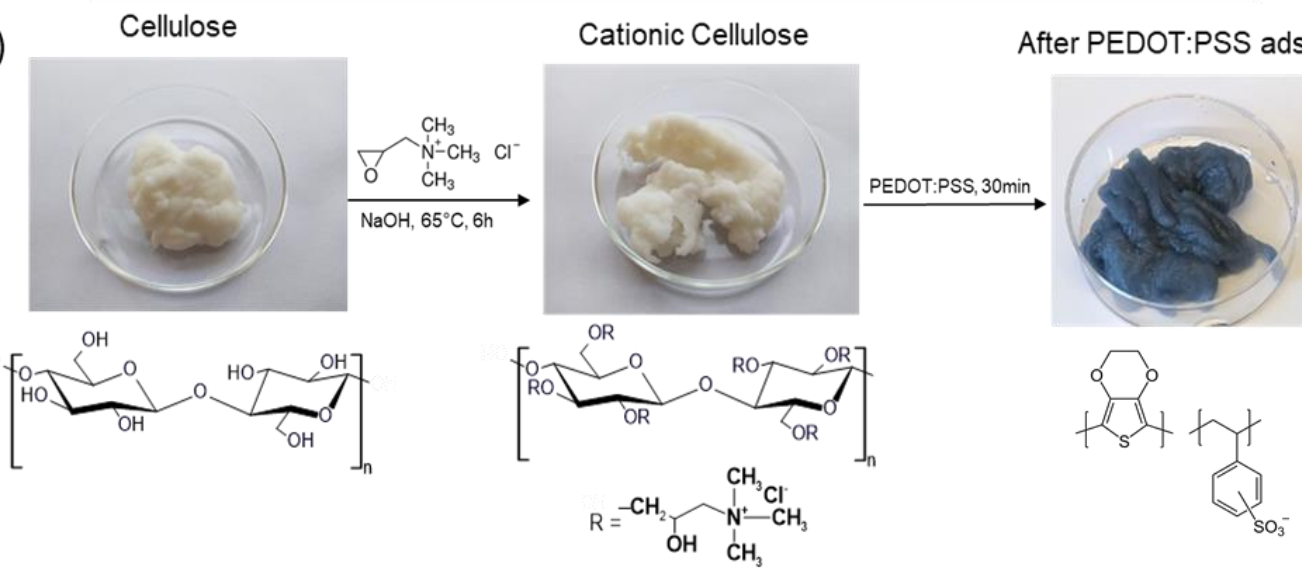

Figure 1. The material concept and process displayed as a visual (a) and the selected papermaking chemistry for the paper machine trials with the cationic pulp preparation and PEDOT:PSS adsorption (b). 


\section{Results \& Discussion}

The work and the results have been divided into different phases. First, pre-trial studies were used to test the material concept on the lab bench. When moving on to the pilot scale, the processability of the material concept was in focus. In-process measurements were combined with structural characterization of the formed papers in order to understand factors affecting the processing of these papers. Then, mechanical and electric characterization of the papers were performed to evaluate the performance of the different formulations and the effect of different additives. Finally, a supercapacitor was assembled and characterized.

\section{Laboratory retention and hand sheet trials}

In order to understand if the different materials could be retained in the paper structure with common retention strategies, retention trials were performed. A cationic polyacrylamide was used as retention aid, and an increased addition of the retention aid resulted in a higher retention of the materials. The increase from $<30 \%$ to $>80 \%$ showed promise that this retention aid also could work in the papermaking process.

The laboratory hand sheets were prepared for electrochemical examination of the combination of cationic pulp fibers, PEDOT:PSS and AC. It was found that adsorbing PEDOT:PSS on the cationic pulp fibers showed a clear effect with halved internal resistance and 17 times increase in areal capacitance. As the capacitance of sheets with only PEDOT:PSS had a negligible capacitance, it could be assumed that it instead plays the role of creating a percolating conducting network along the fibers, which is decisive for exploiting the capacitive property of the loaded AC. This is in line with previously reported work (Belaineh et al 2021), where it was found that $=<10$ wt\% PEDOT:PSS is needed to have an optimum specific capacitance in a system where AC made up the rest of the electroactive material. This could be compared to the present work, where PEDOT:PSS takes up less than $1 \%$ of the total paper weight.

\section{Production of electrode paper rolls on a pilot paper machine}

A $2 \times 2$ experimental matrix was constructed in order to test effects of adding CNF and/or CB to the formulations (table 1). CNF was hypothesized to contribute with improved mechanical properties, and the $\mathrm{CB}$ to contribute with further increased electric conductivity in addition to the PEDOT:PSS.

Table 1. Experimental matrix for the pilot trial, with dry wt\% ratios given for the formulations $A, B, C$ and $D$ with the components cationic cellulose pulp with PEDOT:PSS (CP:PP), activated charcoal (AC), carboxymethylated cellulose nanofibrils (CNF) and carbon black dispersed by CNF (CB:CNF).

\begin{tabular}{c|cc|cc} 
& \multicolumn{2}{|c|}{ Without CNF } & \multicolumn{3}{c}{ With CNF } \\
\hline \multirow{2}{*}{$\begin{array}{c}\text { Without } \\
\text { CB:CNF }\end{array}$} & $\frac{\text { CP:PP }}{51.2 \%}$ & $\frac{\mathrm{AC}}{48.6 \%}$ & $\frac{\mathrm{CP}: \mathrm{PP}}{48.9 \%}$ & $\frac{\mathrm{AC}}{48.6 \%}$ \\
& $\frac{\mathrm{CNF}}{-}$ & $\frac{\mathrm{CB}: \mathrm{CNF}}{-}$ & $\frac{\mathrm{CNF}}{2.3 \%}$ & $\frac{\mathrm{CB}: \mathrm{CNF}}{-}$ \\
\hline \multirow{2}{*}{ With } & $\frac{\mathrm{CP}: \mathrm{PP}}{53.2 \%}$ & $\frac{\mathrm{AC}}{45.9 \%}$ & $\frac{\mathrm{CP}: \mathrm{PP}}{52.2 \%}$ & $\frac{\mathrm{AC}}{44.6 \%}$ \\
CB:CNF & $\frac{\mathrm{CNF}}{-}$ & $\frac{\mathrm{CB}: \mathrm{CNF}}{0.7 \%}$ & $\frac{\mathrm{CNF}}{2.3 \%}$ & $\frac{\mathrm{CB}: \mathrm{CNF}}{0.7 \%}$
\end{tabular}



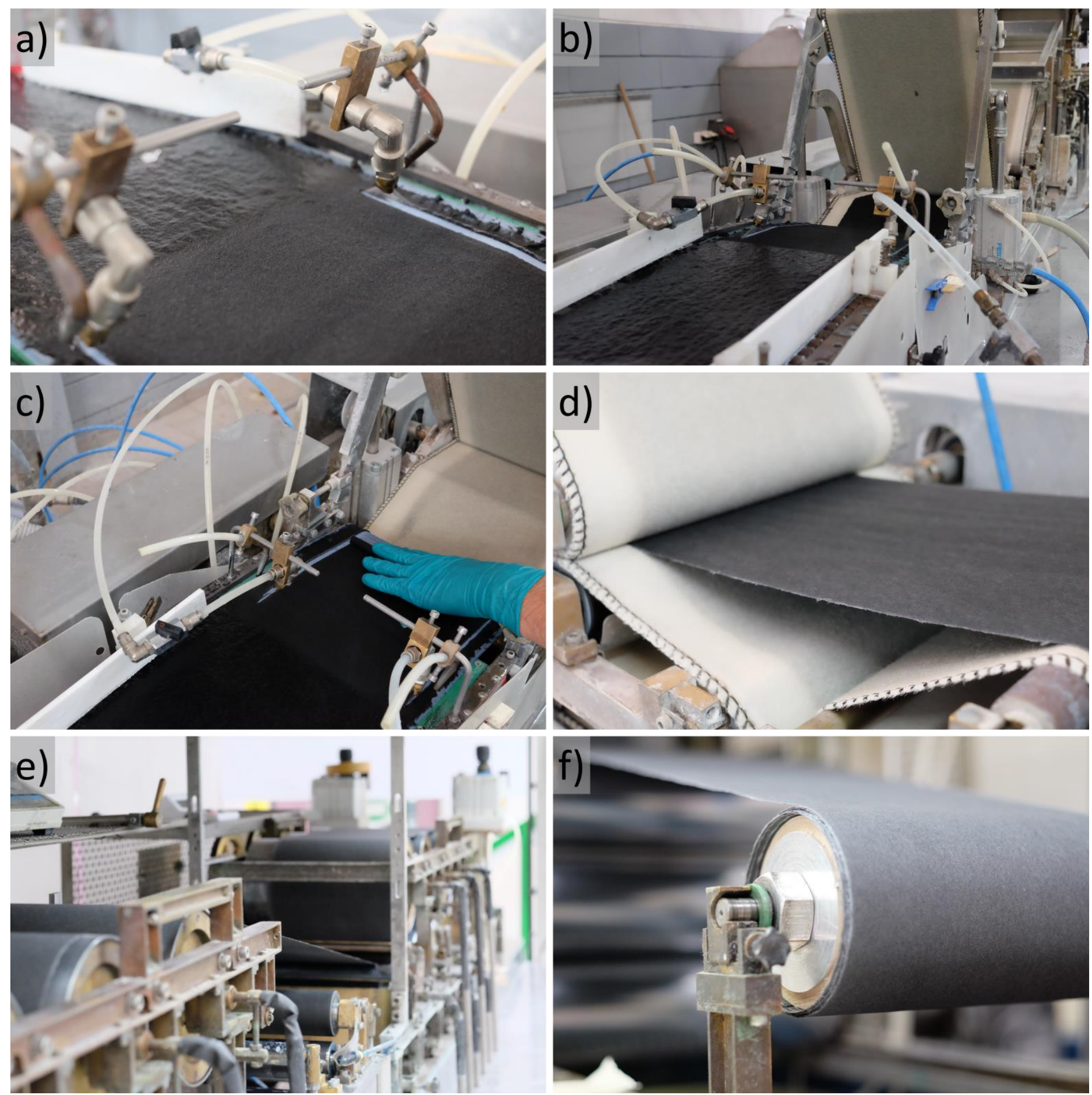

Figure 2. Photographs of the production of the power paper in the paper machine: a) view of the forming section, showing the dry line and the waterjet cutters, b) forming section view towards pressing section, c) manual transfer from the forming to the pressing section, d) paper coming out from the pressing section, e) view from the first drying section towards the second, f) ready electrode paper being winded on the pilot tambour. 
All the four formulations exhibited good runability on the pilot paper machine, where the wet strengths of the papers were well enough for passing from the forming section to the pressing section. One roll of about 10 meters length were produced of each formulation (fig. 2).

As the white water (i.e. paper making filtrate) was colored black, it was clear that the retention was not perfect. As the performance of the composite as a supercapacitor electrode clearly benefits from having a high loading of the active material, a poor retention should be avoided. The TGA results showed a clear difference between samples with and without CNF, where adding CNF to the formulation resulted in a decreased loading of carbonized materials. This points out a poorer retention of the activated carbon in the CNF formulations. The same samples also display bad formation, which is surprising as there commonly exists a tradeoff between good formation and filler retention (Svedberg \& Lindström 2010).

Table 2. Paper composition as determined by TGA, paper formation number derived from B-radiography and wet formulation cationic demands. The systems with CNF stand out with lower carbon loading, poorer retention and a higher cationic demand which relates to an unbalanced charge in the CNF formulations.

\begin{tabular}{|c|c|c|c|c|c|}
\hline & & A & B & C & D \\
\hline $\begin{array}{c}\text { Composition from } \\
\text { TGA }\end{array}$ & Pulp + CNF & $54 \%$ & $62 \%$ & $56 \%$ & $62 \%$ \\
\hline (\% by dry weight) & $A C+C B$ & $46 \%$ & $38 \%$ & $44 \%$ & $38 \%$ \\
\hline \multicolumn{2}{|c|}{ Formation Number } & 12.4 & 17.4 & 12.6 & 15.5 \\
\hline \multicolumn{2}{|c|}{ Cationic demand ( $\mu$ eq. $/ \mathrm{dm}^{3}$ ) } & 4 & 15 & 6 & 12 \\
\hline
\end{tabular}

An explanation could be found in the charge balance of the system. While the $\mathrm{pH}$ in all formulations was stable at pH8, the cationic demand was clearly higher for formulations including CNF (table 2). This clear difference is probably related to the high anionic charge density of CNF which is not compensated by the amount of C-PAM added to the formulations. The negative $\zeta$-potential of AC (-30 mV), confirmed by DLS measurements, thus compete with the CNF in the charge balance and the retention aid from the cationic C-PAM.

In the SEM surface images we can see how this affects the morphology of the papers (fig. 3). The papers with CNF show more aggregated structures of the carbon particles compared to those without CNF. Inadequate dosage of retention aids, increased flocculation and more aggregated filler particles is known to lead to a one-sided filler distribution (Solberg \& Wågberg 2002, Sampson \& Turner 1996). This zdistribution of fillers is observable in paper profiles, why SEM/EDX analysis was performed on crosssections of the papers. On the obtained elemental maps (fig 3i-I), it would have been expected to observe sulfur and nitrogen from PEDOT:PSS and the cationic groups in the pulp fibers, but the low concentrations of the elements was not possible to detect. On the other hand, oxygen and carbon were easily detected which gave clear views of the filler distributions in the papers. The sample with CNF (B) but without CB:CNF indeed stood out with a pronounced skewed concentration profile of AC throughout the Z-direction of the paper (fig. 3j), showing that the filtration retention mechanism dominates in that system. 

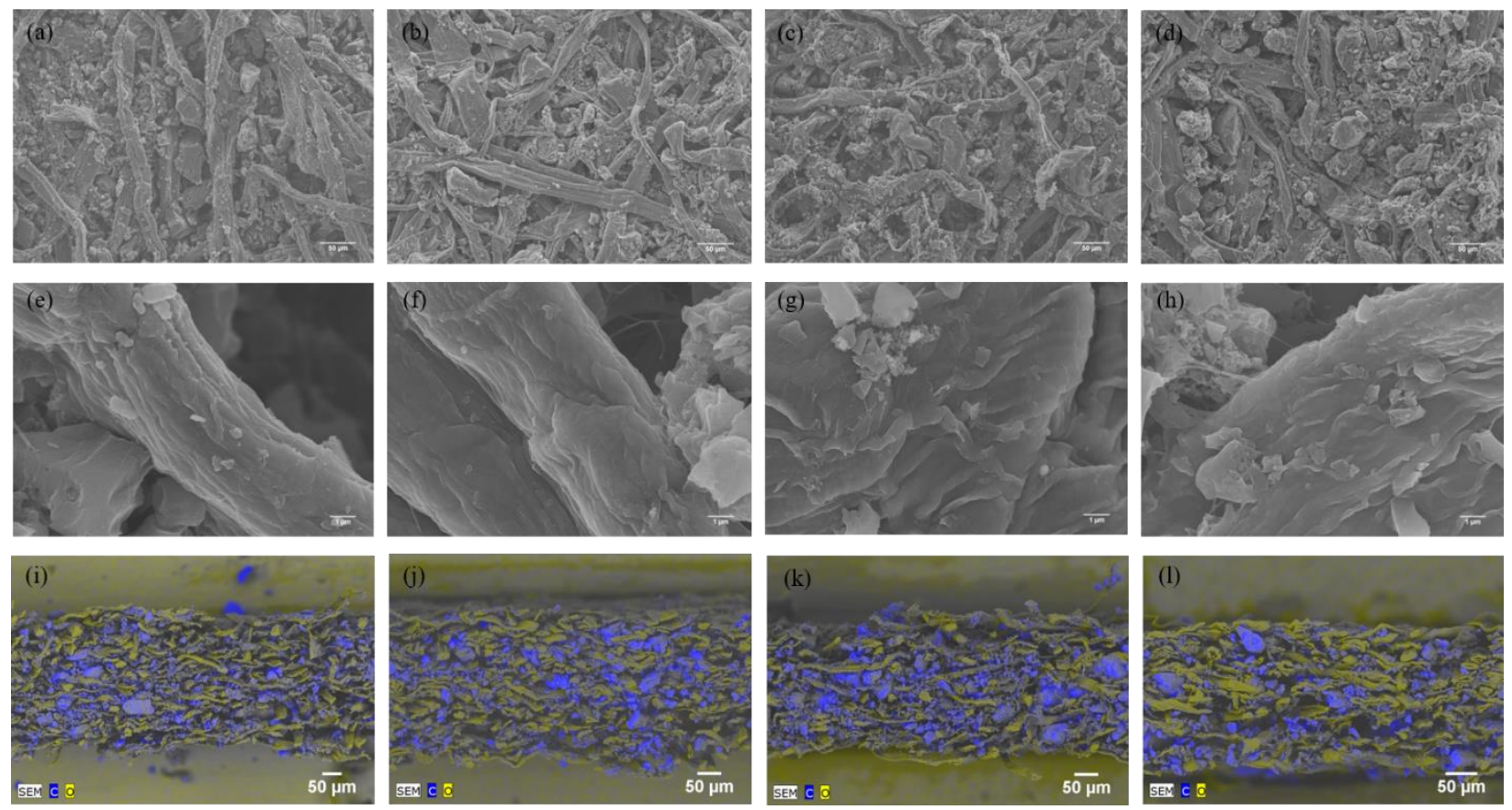

Figure 3. SEM images of papers (top), zoom-in images of fibre surface (middle) and cross-section EDX images of the samples (a), (e), (i) $A,(b),(f),(j) B,(c),(g),(k) C,(d),(f)$, (I) D. On the acquired EDX maps, the oxygen-rich cellulose fibers appear yellow while the carbon-rich $A C$ and $C B$ appear blue.

\section{Mechanical properties}

All the papers had a grammage around $100 \mathrm{~g} / \mathrm{m}^{2}$, but with a varying thickness spanning from 223$265 \mu \mathrm{m}$. All tested mechanical properties did also display a significant effect from CNF, but contrary to the thickness no effect was seen for adding CB:CNF. Both formulations with and without CB:CNF shows more than a doubled tensile strength index as well as tensile stiffness index (fig. 4). This is not consistent with the formation values of the same papers, as papers with poorer formation usually display lower strength (Lindström et al 2005). Further, as these papers are bulkier, they should be weaker due to the reduced number of fiber joints. This should support the assumption that the CNF is useful as a dry-strength agent and filler containment, which is in line with previous reports of adding cellulose nanofibrils to paper formulations (Osong et al 2014). The lower loading of AC in the CNF-containing papers should however also have better mechanical properties due to the inverse relationship between filler content and both tensile strength and tensile stiffness (Tanaka et al 2001).

a

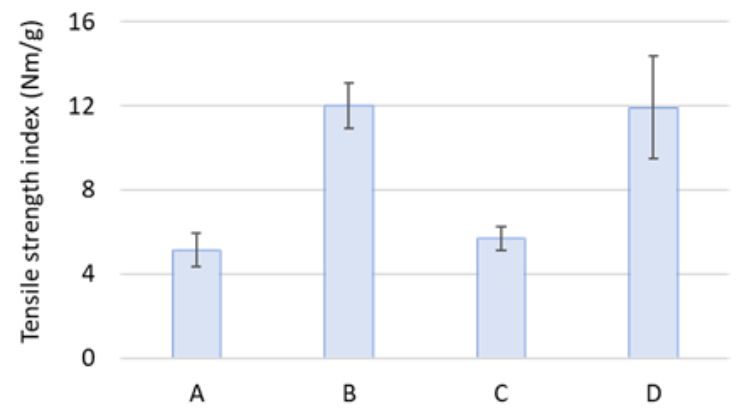

b

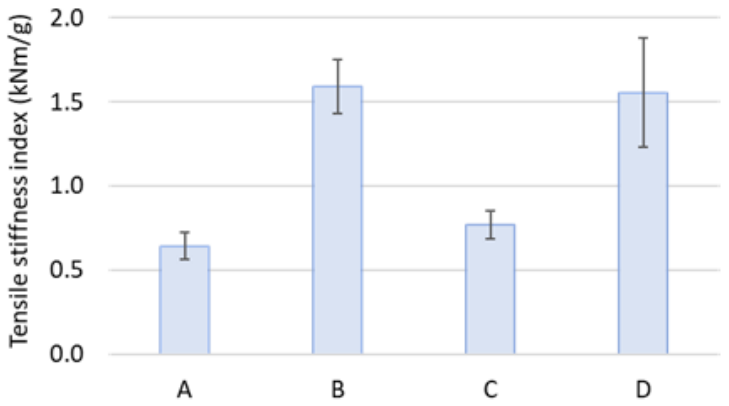

Figure 4. (a) Tensile strength index and (b) tensile stiffness index for the different paper samples. A significant effect can be seen for adding CNF to the formulations. 


\section{Electrical characterization}

The conductivities of all the formed papers were in the magnitude of about $1-10 \mathrm{~S} / \mathrm{m}$, although significant differences were seen between the papers. The samples with CNF had a negative effect on the conductivity, while CB:CNF increased the conductivity (fig. 5a). Nanofibrillated celluloses such as CNF is known to form insulating barriers between the conductive particles (Andres et al 2018), but no such phenomena can be observed in the SEM images. An alternative explanation could be derived from a poorer percolating network following the aggregation of the carbons in the CNF systems combined with the lower loading of AC in the same samples. Regardless of explanation, however, the additives show significant effects although the absolute increase or decrease in conductivity are just a matter of some few $\mathrm{S} / \mathrm{m}$.

The measured specific capacitance of the entire paper ranged from 26 to $30 \mathrm{~F} / \mathrm{g}$, where the papers with less loading of AC displayed a lower capacitance. However, if the specific capacitance is normalized to the loading of AC (i.e. normalized to the electroactive material mass), no difference could be seen between the different samples. The internal resistance did not significantly differ between the papers (fig. $5 \mathrm{c}$ ), thus, the CNF and/or CB:CNF additions to the formulations were not found to affect the electrochemical performance of the $A C$ in this material system.

a

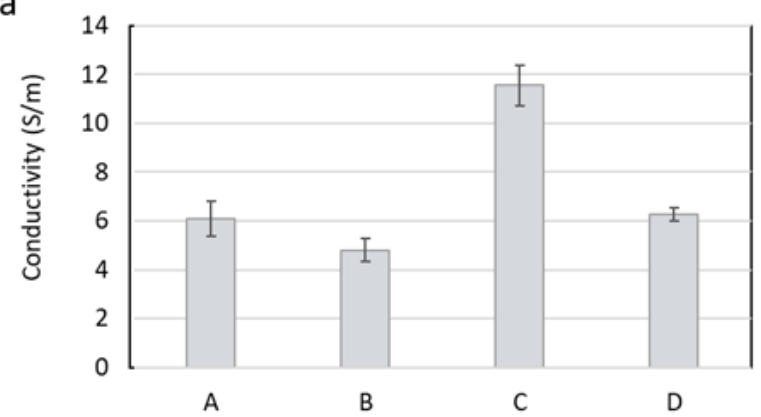

C

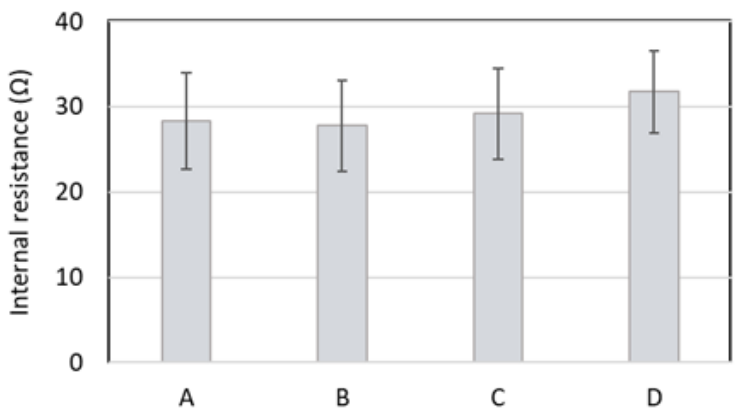

b

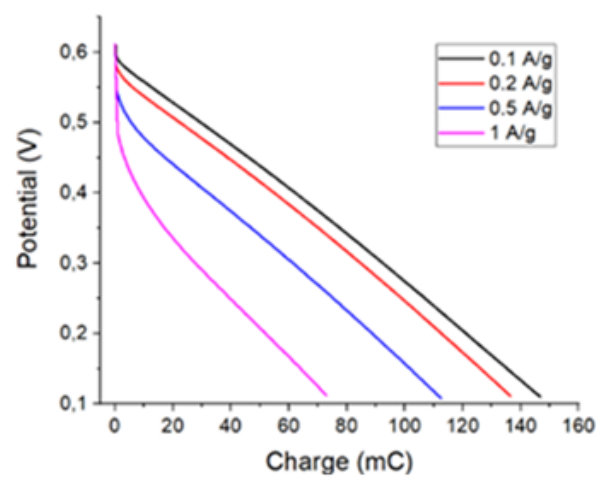

d

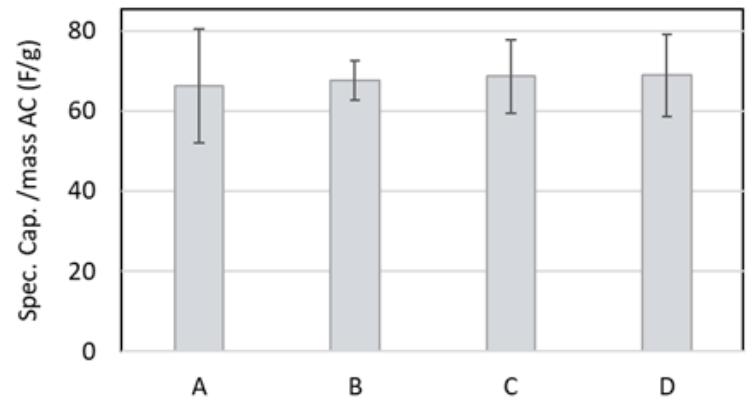

Figure 5. The conductivity differs significantly between the samples (a), whereas galvanostatic discharge measurements (b) reveals no significant difference neither for the internal resistance (c) nor for the AC specific capacitance. The discharge at different current levels displayed in (b) is for paper A.

The specific capacitance of the paper electrodes is in the order of commercial supercapacitor electrodes (Obreja et al 2010), still the capacitance could be considerably higher. More than exploring strategies to load more than around $40 \mathrm{wt} \% \mathrm{AC}$ in the web, the AC itself could be better utilized. The average achieved specific capacitance of the AC in this work is about $68 \mathrm{~F} / \mathrm{g}$ (fig. $5 \mathrm{~d}$ ). This can be compared to other reported works where similar grades of AC from the same supplier have been used as references, where the AC reaches specific capacitances ranging up to 160-180 F/g (Li et al 2013, Wang et al 2015). 
As the conductivity is significantly affected both by CNF and CB:CNF with no corresponding change in the specific capacitance, this points out that the in-plane conductivity is not the limiting factor at this stage. Continued development of AC-loaded papers for double-layer charge storage should therefore investigate other potentially limiting factors.

Nevertheless, the formed paper electrodes can store significant amounts of charge without being produced in a setting focused on optimization. The achieved specific capacitance of the AC in this work is about 65-70 F/g, which is a good value compared to the performance of materials in commercial supercapacitors (Obreja et al 2010). Another important feature with these paper electrodes is the potential to have thick electrodes with preserved AC-electrolyte contact, as the cellulose fibers themselves swells in the electrolyte and provides a network for electrolyte supply. The present paper electrodes have a grammage around $100 \mathrm{~g} / \mathrm{m}^{2}$, but could theoretically be made several times thicker.

To demonstrate the applicability of these papers as supercapacitor electrodes, an electrolytic doublelayer supercapacitor device was constructed (fig. 6). Although an aqueous $\mathrm{KCl}$ electrolyte is not preferable in a device setting given the limited potential range and corrosion on the aluminum current collector used, $\mathrm{KCl}$ was used to enable a comparison with the half-cell measurements. It was found that the specific capacitance was clearly affected by the discharge current (fig. 6c), where a lower current level gave a higher measured capacitance. Although the achieved capacitance is lower than in the 3-electrode setup, this demonstrates that the paper can function as an electrode in a supercapacitor.

a

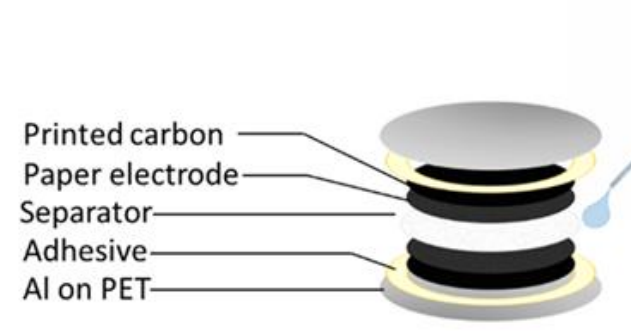

C

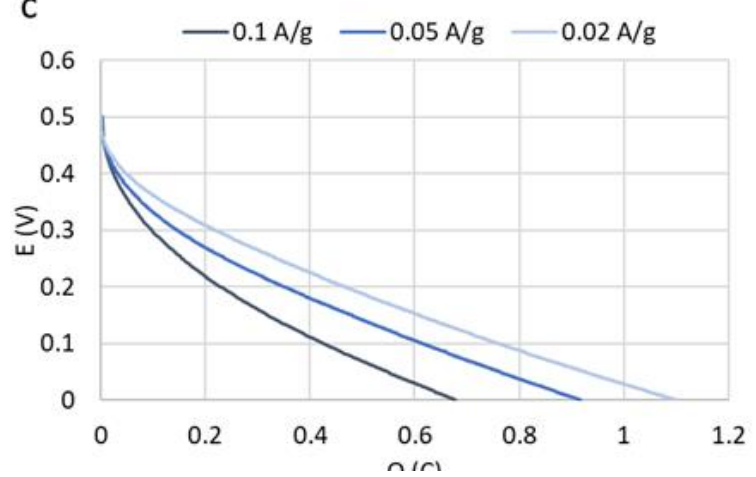

b
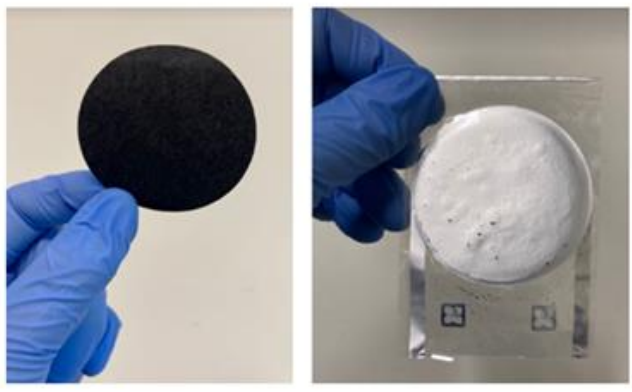

$\mathrm{d}$

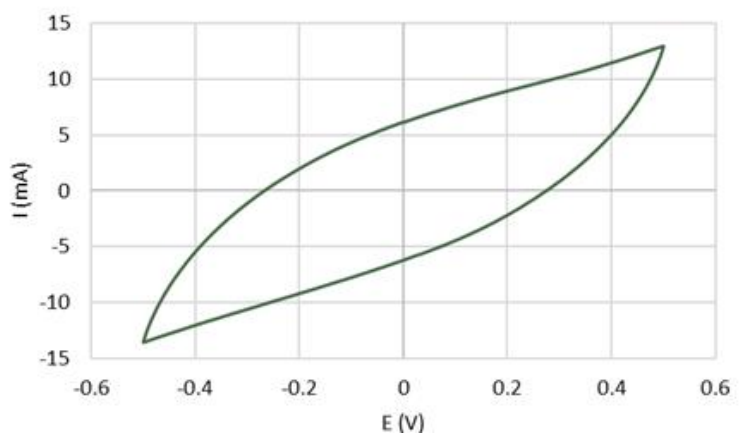

Figure 6. (a) Schematic of the supercapacitor device, (b) photos of the cut electrode paper and the assembled device, (c) discharge at different discharge currents and (d) cyclic voltammetry at $10 \mathrm{mV} / \mathrm{s}$ scan rate. 


\section{Conclusions}

Electrode papers for an energy storage application were produced in a pilot-scale paper machine, with up to $99 \%$ of bio-based and $100 \%$ organic materials used in the formulations. Pre-trial laboratory hand sheet studies demonstrated that a combination of cationized dissolving pulp with very small amounts of electrostatically adsorbed PEDOT:PSS can create a conducting percolating network to connect the chargestoring activated charcoal (AC) particles.

The material concept was successfully transferred to pilot paper making conditions, where four different formulations were designed for the investigation of the effects for adding cellulose nanofibrils (CNF) and/or conductive carbon black (CB). The addition of CNF was shown to lower the retention of $A C$ and $C B$, thus resulting in a lower capacitance per area. The poorer retention is likely caused by insufficient charge balancing in the systems when adding highly anionic CNF to formulations without adjusting the dosage of the cationic retention agent. On the other hand, it is believed that the CNF is important for improving the mechanical properties, although the contribution from CNF is not possible to quantify as the same papers also had lower filler contents (which often results in better mechanical performance).

The addition of CB and CNF had a significantly effect on the in-plane conductivity of the papers, where and increase in conductivity was observed when adding CB while the addition of CNF decreased conductivity. However, the specific capacitance (calculated per-mass-unit of $A C$ ) was neither affected by the addition of CNF nor CB to the formulations, and displayed an average specific capacitance of about $70 \mathrm{~F} / \mathrm{g}$.

The electrode specific capacitance of about $30 \mathrm{~F} / \mathrm{g}$ is about the same order of commercial supercapacitor electrodes. This, in combination with the good runability and descent mechanical properties, demonstrates the feasibility of using paper making techniques for fabricating supercapacitor electrodes. Furthermore, this work expands the knowledge about critical aspects in the design of the formulations and process design, and advances the technological frontier of paper-based energy storage solutions.

\section{Experimental section}

\section{Materials}

\section{Preparation of cationic pulp}

To prepare cationically charged cellulose fibres, quaternary trimethylammonium groups are grafted on cellulose (fig. 1). The reaction was performed according to the procedure by Zhou et al (2013) with some modifications. Briefly, 400g (dry weight) of a softwood sulfite dissolving pulp (Domsjö Dissolving plus, Domsjö Fabriker AB, Sweden) was mixed with 4.78M NaOH (Sodium Hydroxide pellets, VWR) and left for $10 \mathrm{~min}$. Afterwards, 612 $\mathrm{g}$ of isopropanol and 400g of glycidyl trimethylammonium chloride (GTMAC, 99.9\% purity, Sigma Aldrich) were added and the reaction mixture was mixed thoroughly.

The reaction was carried out at $65 \stackrel{\circ}{ } \mathrm{C}$ for 6 hours in a hot water bath. The plastic bag with reaction mixture was taken out every $1 \mathrm{~h}$ to knead the bag in order to mix the pulp for homogeneous reaction. The reaction mixture was then neutralized with hydrochloric acid, filtered and washed thoroughly with deionized water. In total $2 \mathrm{Kg}$ of the cationic pulp was prepared for the paper machine. The obtained charge density of pulp, measured with polyelectrolyte titration, was $250 \pm 50 \mu \mathrm{eq} / \mathrm{g}$. 
Adsorption of PEDOT:PSS to pulp

PEDOT:PSS saturated fibres were prepared according to the following procedure (fig. 1). A fibre dispersion at $2 \mathrm{~g} / \mathrm{L}$ was mixed with PEDOT:PSS (10g/L, Agfa) dispersion and stirred for $30 \mathrm{~min}$. The fibres were then filtered and washed thoroughly with deionized water to remove unadsorbed PEDOT:PSS. The adsorbed amount of PEDOT:PSS was calculated based on concentration of PEDOT:PSS left in the solution after saturation. The amount adsorbed was 0.75-1.5 wt\% PEDOT:PSS.

\section{Preparation of CNF-Carbon}

The preparation of the carboxymethylated Cellulose NanoFibrils (CNF) and Carbon Black mixture is presented below. The CNF was produced from a softwood sulphite dissolving pulp (Domsjö Dissolving plus) from Domsjö Fabriker AB, Sweden. The pulp was carboxymethylated to a degree of substitution (DS) of 0.1, see Wågberg et al. (1987) for details. After the carboxymethylation, the pulp was homogenized (Microfluidizer M-110EH, Microfluidics Corp., USA) one time at 1700 bar at 2 wt\%, by two serial reaction chambers with smallest dimension $200 \mu \mathrm{m}$ and $100 \mu \mathrm{m}$, respectively. Carbon black was acquired from Fisher Scientific with the brand name: Thermo Scientific Alfa Aesar 039724.A1. The CNF:CB (1:10) mixture was prepared by adding $250 \mathrm{~g} \mathrm{CB}$ to $1250 \mathrm{~g}$ CNF at 2 wt\% and $4.75 \mathrm{~g}$ deionized water, where after the mixture was passed through the above-mentioned homogenizer at 400 bar and two serial chambers with smallest dimension of $400 \mu \mathrm{m}$ and $200 \mu \mathrm{m}$, respectively.

\section{Materials used as received}

Activated charcoal (AC) made from steam activated wood charcoal (Norit ${ }^{\circledR}$ A Supra, Acros Organics) was selected as the main charge storage material. Cationic polyacrylamide (C-PAM) was selected as retention aid. For the lab trials, Eka PL 1510 CPAM was used, and a corresponding C-PAM (Kemira Fennopol K4230 T) was used in the pilot trials.

\section{Paper production}

Retention laboratory trials

Retention trials were performed in a Britt Dynamic Drainage Jar and a standard screen with pore size of 76 $\mu \mathrm{m}$ (125P). The pulp was diluted to $5 \mathrm{~g} / \mathrm{L}$ by mixing at $1000 \mathrm{rpm}$ and a volume of $500 \mathrm{ml}$ was used. The additional components were added sequentially with $30 \mathrm{~s}$ interval in the order of AC, CNF:CB and lastly CPAM 1510. A $100 \mathrm{ml}$ sample was taken after an additional $30 \mathrm{~s}$ and the concentration was determined and compared with the added material to result in a retention value.

Hand sheets

To test the effect from PEDOT:PSS on the performance of the system before moving to pilot scale, laboratory hand sheets were prepared using a Rapid Köthen sheet former with automatic couching and drying. Three hand sheets were prepared; cationic pulp with PEDOT:PSS only, cationic pulp without PEDOT:PSS but with AC and thirdly all materials combined. The laboratory hand sheets were evaluated electrochemically as described below.

\section{Paper roll production}

The pilot trial was designed as a factorial experiment, where a matrix of four different formulations was used to determine the effects of added CNF and/or CB (table 1). All four formulations were based on AC and PEDOT:PSS-adsorbed cationic pulp (CP:PP), why the first formulation consisted only of these components to act as a reference. The second formulation was chosen to include CNF, a third one including CNF:CB and the fourth contained both CNF and CNF:CB. All formulations included 0.2 wt\% C-PAM as a retention agent. 
The pilot production of the electrode papers was carried out on a pilot-scaled, $20 \mathrm{~cm}$-wide fully equipped paper machine at Ahlstrom-Munksjö's R\&D center located in Apprieu, France. The different wet-end formulations were prepared in chests before being pumped into the headbox at a consistency in the range of $6-6.7 \mathrm{~g} /$ liter. The web speed was set to 1 meter/minute, a speed which allows orientation of the fibers while allowing manual handling of the paper along the machine. Edges were trimmed in the forming section using waterjet cutting. The web was manually passed from the forming section to the two pressing nips (each set to $2 \mathrm{~kg}$ ) and subsequent drying sections. The first drying section held a temperature between 138$180^{\circ} \mathrm{C}$ and the second drying section between $124-155^{\circ} \mathrm{C}$. The formed paper, with a moisture content of about $6 \%$, was collected as 10 -meter-long webs winded on rolls.

\section{Material characterization}

\section{Thermogravimetric analysis}

Differential thermogravimetric analysis (TGA) was performed using a Mettler Toledo DSC1 in ambient atmosphere and a heating rate of $20^{\circ} \mathrm{C} / \mathrm{min}$. Reference measurements on the PEDOT:PSS-adsorbed cationic pulp, $\mathrm{AC}$ and $\mathrm{CB}: \mathrm{CNF}$ were used to calculate the different components in the formed papers, by exploiting the different thermal degradation patterns of cellulose and carbonized materials (Isacsson et al 2020).

\section{Scanning electron microscopy}

Hitachi S-4800 Field-Emission Scanning Electron Microscope (FE-SEM) was used for studying paper rolls surfaces. All the samples were coated with Pt/Pd to avoid charging in SEM. Cross-section samples of the paper cut perpendicular to the machine direction were prepared using a Leica histological microtome. The cross-sections were imaged using a scanning electron microscope (SEM) (Zeiss, EVO50 LaB6Tip) and an energy-dispersive X-ray spectroscopy (EDX) (Bruker, Quantax 200 SDD $30 \mathrm{~mm} 2$ ). On the obtained X-ray maps, oxygen was used as a marker for the pulp fibers, and carbon was used as a marker for the activated carbon.

\section{Mechanical testing and formation}

The mechanical properties were determined in the machine direction (MD) according to ISO1924-3 and the thickness according to SCAN-P 88:01. The formation was determined by the method based on $\beta$ radiography (Johansson and Norman, 1996).

\section{Dynamic Light scattering (DLS)}

Zeta potential measurements were performed using dynamic light scattering (DLS) with backscattering detector (Zetasizer ZEN3600, Malvern Instruments Ltd. U.K.).

\section{Conductivity}

Electrical sheet resistance was measured using a four-point probe system (Ossilla). For the hand sheets, each sample was probed and measured at three different points close to the center of the sample. For the machine-produced papers, a $6 \mathrm{~cm}$ wide cross strip of each paper was collected on which three probing positions was used; left edge, center and right edge in order to investigate any cross-direction effect on the conductivity. For each position, three measurement points were used.

The sample thickness was measured using a micrometer caliper. An average thickness, taken from three different points, was used together with the sheet resistance measurements to calculate the sample inplane conductivity. 


\section{Electrochemical characterization}

A $1 \times 1 \mathrm{~cm}$ patch was cut out for each sample for electrochemical characterization. Wet-proofed (polytetrafluoroethylene-impregnated) polyacrylonitrile carbon fiber sheets (Toray carbon paper) were chosen as a conducting support for the patches. The carbon paper was taped on one side with polyimide (Kapton) tape for improved mechanical stability, after which $1 \mathrm{~cm}$ wide supports were prepared from the taped sheets. The sample patches were mounted on individual supports by gluing them on the tape-free side using a carbon ink, cured on a hot plate $\left(150^{\circ} \mathrm{C}\right)$ for 10 minutes.

The electrochemical characterizations were performed with a 3-electrode setup in a cell containing 20ml $1 \mathrm{M}$ potassium chloride, with galvanized steel mesh as counter electrode and an $\mathrm{Ag} / \mathrm{AgCl}$ reference electrode. A potentiostat (Ivium Octostat5000) was used along with Ivium potentiostat software. For each characterization, the open circuit potential (OCP) vs $\mathrm{Ag} / \mathrm{AgCl}$ as used as the starting point for the measurements.

Galvanostatic charge/discharge measurements were carried out with a set of current levels for one of the samples, after which every sample were investigated at current of $0.9 \mathrm{~mA}$, with a voltage maximum of OCP $+0.5 \mathrm{~V}$. Cyclic voltammetry was done in the potential window of OCP $+/-0.5 \mathrm{~V}$ at a scan rate of $10 \mathrm{mV} / \mathrm{s}$.

\section{Statistical analysis}

Two-way analysis of variance (ANOVA) in order to determine both separate and combined effects of CNF and CNF:CB addition. As papers are rather heterogenous systems, a significance level of $\alpha=0.05$ was set to determine if the null hypothesis should be rejected. The analysis was performed assuming a normal distribution of the data.

\section{Device assembly and characterization}

Circular electrodes $(\varnothing=5.5 \mathrm{~cm})$ of the $A C+C P+P: P+C B: C N F$ sample as well as circular separators from cleanroom wipes $(\varnothing=6.0 \mathrm{~cm})$ were prepared by laser cutting (details?). The laser-cut patches were mounted on the carbon-printed collectors prepared in previous work (Say et al 2020) by gluing them using a commercial carbon ink (7102 Carbon Conductor, DuPont), which then was cured on a hot plate $\left(100^{\circ} \mathrm{C}\right)$ for 30 minutes.

A ring of double-sided tape was mounted around the glued papers on both collectors. The papers were soaked with $1 \mathrm{M} \mathrm{KCl}(\mathrm{aq})$, after which a cleanroom wipe was mounted on top of one of the papers, partially overlapping the tape. The structure was then laminated and sealed by mounting the separator-free side on top of the separator and the double-sided tape (fig. 6). The assembled device was electrochemically characterized using an Ivium Octostat5000 potentiostat along with Ivium potentiostat software. Cyclic voltammetry was done in the potential window of $\mathrm{OCP}+/-0.5 \mathrm{~V}$ at $10 \mathrm{mV} / \mathrm{s}$ scan rate.

\section{Acknowledgements}

This work was funded by the Digital Cellulose Centre, a competence center set up by the Swedish Innovation Agency VINNOVA (2016-05193) and a consortium of Swedish industries, as well as the Swedish Foundation for Strategic Research (GMT14-0058). As contributions to the Digital Cellulose Center, Agfa has kindly supplied PEDOT:PSS and Ahlstrom-Munksjö has kindly put their pilot paper machine to the project's disposal. We also acknowledge the support from Treesearch, a collaboration platform for Swedish forest industrial research. 


\section{References}

Andres, Britta, et al. "Cellulose binders for electric double-layer capacitor electrodes: The influence of cellulose quality on electrical properties." Materials \& Design 141 (2018): 342-349.

Belaineh, D., Brooke, R., Sani, N., Say, M. G., Håkansson, K. M. O., Engquist, I., Berggren, M. and Edberg, J. (2021) Printable carbon-based supercapacitors reinforced with cellulose and conductive polymers [Manuscript submitted for publication]

Brooke, R., Edberg, J., Say, M. G., Sawatdee, A., Grimoldi, A., Åhlin, J., ... \& Engquist, I. (2019). Supercapacitors on demand: all-printed energy storage devices with adaptable design. Flexible and Printed Electronics, 4(1), 015006.

Bubnova, O. (2021). A decade of R2R graphene manufacturing. Nature nanotechnology, 16(10), 10501050.

Isacsson, P., Wang, X., Fall, A., Mengistie, D., Calvie, E., Granberg, H., Gustafsson, G., Berggren, M. \& Engquist, I. (2020). Highly Conducting Nanographite-Filled Paper Fabricated via Standard Papermaking Techniques. ACS applied materials \& interfaces, 12(43), 48828-48835.

Johansson, P.-Å., and Norman, B. (1996) Methods for evaluating formation, print unevenness and gloss variations developed at STFI. 1996 Process and product quality conference, 14-17 October, Cincinnati, $\mathrm{OH}$, USA, pp. 139-145.

Li, Z., Xu, Z., Tan, X., Wang, H., Holt, C. M., Stephenson, T., ... \& Mitlin, D. (2013). Mesoporous nitrogen-rich carbons derived from protein for ultra-high capacity battery anodes and supercapacitors. Energy \& Environmental Science, 6(3), 871-878.

Lindström, T., Wågberg, L., \& Larsson, T. (2005, September). On the nature of joint strength in paper-A review of dry and wet strength resins used in paper manufacturing. In 13th fundamental research symposium (Vol. 1, pp. 457-562). Cambridge, UK: The Pulp and Paper Fundamental Research Society.

Malti, A., Edberg, J., Granberg, H., Khan, Z. U., Andreasen, J. W., Liu, X., ... \& Berggren, M. (2016). An organic mixed ion-electron conductor for power electronics. Advanced science, 3(2), 1500305.

Obreja, V. V., Dinescu, A., \& Obreja, A. C. (2010). Activated carbon based electrodes in commercial supercapacitors and their performance. Int. Rev. Electr. Eng, 5(1).

Osong, S. H., Norgren, S., \& Engstrand, P. (2014). Paper strength improvement by inclusion of nanolignocellulose to Chemi-thermomechanical pulp. Nordic Pulp \& Paper Research Journal, 29(2), 309-316.

Pei, A., Butchosa, N., Berglund, L. A., \& Zhou, Q. (2013). Surface quaternized cellulose nanofibrils with high water absorbency and adsorption capacity for anionic dyes. Soft Matter, 9(6), 2047-2055.

Peng, Y., Gardner, D. J., \& Han, Y. (2012). Drying cellulose nanofibrils: in search of a suitable method. Cellulose, 19(1), 91-102.

Sampson, W. W., \& Turner, P. J. (1996). The effect of suspension crowding on the in-plane distribution of filler in handsheets. Appita journal, 49(4), 274-276.

Say, M. G., Brooke, R., Edberg, J., Grimoldi, A., Belaineh, D., Engquist, I., \& Berggren, M. (2020). Spraycoated paper supercapacitors. npj Flexible Electronics, 4(1), 1-7. 
Solberg, D., \& Wågberg, L. (2002). On the mechanism of GCC filler retention during dewatering - New techniques and initial findings. Journal of Pulp and Paper Science (JPPS), 28(6), 183-188.

Svedberg, A., \& Lindström, T. (2010). The effect of various retention aids on retention and formation. Nordic Pulp \& Paper Research Journal, 25(2), 195-203.

Tanaka, A., Niskanen, K., Hiltunen, E., \& Kettunen, H. (2001). Inter-fiber bonding effects of beating, starch or filler. Nordic Pulp \& Paper Research Journal, 16(4), 306-312.

Wang, D., Geng, Z., Li, B., \& Zhang, C. (2015). High performance electrode materials for electric double-layer capacitors based on biomass-derived activated carbons. Electrochimica Acta, 173, 377-384.

Wang, Z., Lee, Y. H., Kim, S. W., Seo, J. Y., Lee, S. Y., \& Nyholm, L. (2021). Why cellulose-based electrochemical energy storage devices?. Advanced Materials, 33(28), 2000892.

Wågberg, L., Winter, L., Ödberg, L., \& Lindström, T. (1987). On the charge stoichiometry upon adsorption of a cationic polyelectrolyte on cellulosic materials. Colloids and Surfaces, 27(4), 163-173. 